\title{
Intraocular lens power calculation for emmetropia: a clinical study
}

\author{
JEFFREY S. HILLMAN \\ From the St James's University Hospital, Leeds
}

SUMMARY A series of 50 eyes received an intraocular lens (IOL) of power calculated for emmetropia from data of axial length, corneal curvature, and postoperative anterior chamber depth by R. D. Binkhorst's formulae. The postoperative refraction results were compared with those of 100 control eyes which received $+19 \mathrm{D}$ standard power IOLs without calculation. The calculated group had postoperative refractions which were closer to emmetropia, and the difference was of statistical significance, with $92 \%$ within the $\pm 1 \mathrm{D}$ range and $98 \%$ within the $\pm 2 \mathrm{D}$ range from emmetropia. The calculated predictions of postoperative refraction were of a useful level of accuracy. Consideration of the sources of error indicates that there is no justification for the use of IOLs in power steps of less than $1 \mathrm{D}$. The calculation of IOL power allows the surgeon to control the postoperative refraction and avoid unwanted ametropia.

The intraocular lens (IOL) is being used in the surgical management of cataract by an increasing number of British surgeons because of the high quality of resulting vision without demands on the patient. The quality of postoperative vision depends to a degree on the postoperative refractive error. Postoperative astigmatism may be controlled by careful surgical technique with particular attention to suture placement and tension. The residual spherical error is a.function of the basic refractive power of the aphakic eye and the power of the IOL which is implanted. The use of IOLs in a standard power gives a satisfactory postoperative refraction in a large percentage of cases, but there remain a number of eyes with unplanned postoperative ametropia. The use of IOLs of different powers selected after calculations made preoperatively from data of ocular dimensions offers a way of controlling the postoperative refraction.

A previous study' showed that the cautious use of IOLs in a narrow range of powers around the standard power does not significantly influence postoperative ametropia compared with the use of IOLs of standard power. It was suggested that this is because ametropia occurs in eyes with abnormal ocular dimensions which need IOLs of more extreme powers. This paper reports a prospective study investigating the degree of

Correspondence to J. S. Hillman. FRCS, Department of Ophthalmology, St James's University Hospital, Leeds LS9 7TF. control over postoperative refraction which can be obtained by the use of IOLs in a wide range of powers as calculated to give emmetropia.

\section{Material and methods}

The study was conducted on 150 eyes undergoing cataract extraction with implantation of a Binkhorst iris-clip IOL. The control population of 100 eyes received an IOL of standard $+19 \mathrm{D}$ power. Forty-two patients were male, 52 were female, and 6 had bilateral surgery. Their mean age $( \pm S D)$ was $67 \cdot 4 \pm$ 14.5 years, and the range of preoperative refractions of the eyes is shown in Fig. 1.

The study population consisted of 50 eyes in 18 males and 32 females which received an IOL of power the nearest whole dioptre to that calculated to give emmetropia. They were of mean age $( \pm S D) 71 \cdot 4 \pm 7 \cdot 3$ years and the range of preoperative refractions shown in Fig. 1 indicates that the group is comparable with the control group.

The calculation of IOL power was based on data of corneal curvature, axial length, and a figure for the postoperative anterior chamber depth. Corneal radius of curvature was taken as the average of measurements in 2 meridia with a Haag-Streit keratometer. Axial length was measured with a Kretz 7200 MA ophthalmic A-scan ultrasound instrument with a $10 \mathrm{MHz}$ transducer. The probe was coupled to 
Fig. 1 The preoperative refractions (spherical equivalent) for the control and calculated IOL groups.

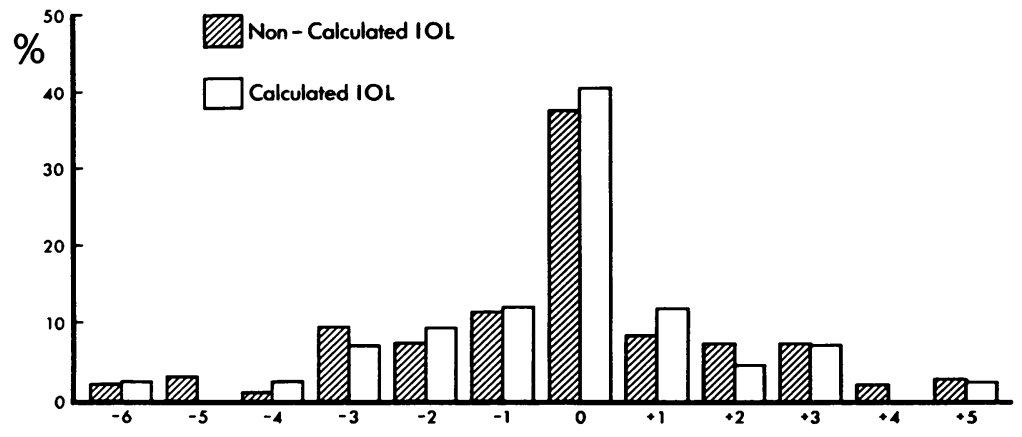

the anaesthetised eye by $5 \%$ methylcellulose solution in a contact lens water bath. Measurements were obtained from Polaroid photographs taken when axial alignment of the ultrasound beam was indicated by high echo peaks from the cornea, both surfaces of the lens, and the vitreoretinal interface. The measurement scale in the instrument was calibrated for the axial length to be read directly off the photographs in $\mathrm{mm}$, on the assumption of a hypothetical common speed of 1550 metres/second for ultrasound in ocular tissues. To allow for several factors which tend to give undermeasurement a correction factor of $0.25 \mathrm{~mm}$ was added to the axial length measurement. The figure of $3.19 \mathrm{~mm}$ was taken as the distance from the vertex of the cornea to the anterior vertex of the IOL, as this is an accepted figure for the style of IOL used.

Calculation was performed by a Wang 2200T computer by means of Binkhorst's formulae. ${ }^{2}$ The first formula gave the IOL power for postoperative emmetropia:

$$
D=\frac{1336(4 r-a)}{(a-d)(4 r-d)}
$$

$\mathrm{D}=$ power of IOL in aqueous (dioptres); $\mathrm{r}=$ corneal radius $(\mathrm{mm})$; $a=$ axial length $(\mathrm{mm}) ; \mathrm{d}=$ postoperative anterior chamber depth plus corneal thickness.
The second formula gave a prediction of the postoperative refraction to be expected with any stated power of IOL:

$$
R_{S}=\frac{1336(4 r-a)-D(a-d)(4 r-d)}{1336[v(4 r-a)+0 \cdot 003 a r]-D(a-d)[v(4 r-d)+0 \cdot 003 d r]} .
$$

$\mathbf{R}_{\mathrm{S}}=$ spectacle refraction (dioptres); $\mathbf{v}=$ back vertex distance (metres).

Cataract extraction was personally performed by a microsurgical technique with general anaesthesia and hyperventilation. Limbal incision was made ab externo under a limbal-based conjunctival flap. After a single peripheral iridectomy $\alpha$-chymotrypsin was instilled and 8/0 virgin silk sutures (usually 5) inserted across the wound. The lens was extracted by cryoprobe and acetycholine instilled to constrict the pupil and reconstitute the anterior chamber. A RaynerBinkhorst iris-clip lens was inserted by the closedchamber technique, with avoidance of corneal contact. A loose 10/0 Ethilon safety-sling suture was placed through the upper anterior loop of the IOL and the margin of the peripheral iridectomy at the junction of the outer and middle thirds of the iris. The wound was closed with particular attention to suture tension to minimse induced astigmatism. The post-
Fig. 2 The postoperative refractions (spherical equivalent) for the control and calculated IOL groups.

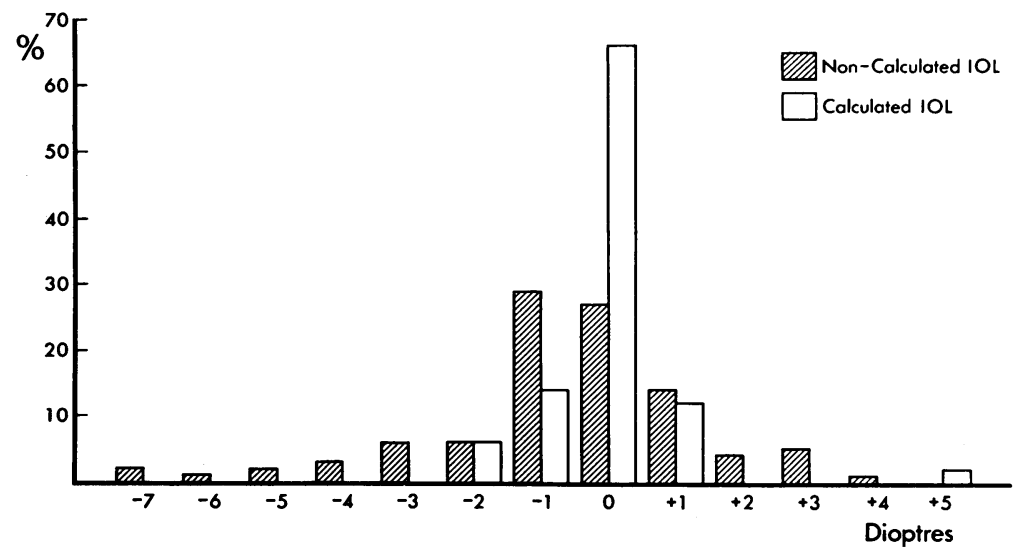




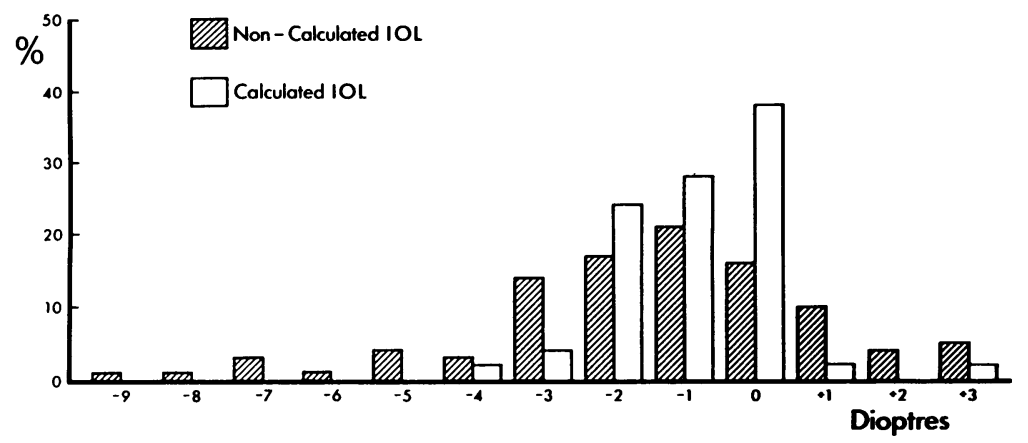

Fig. 3 The postoperative refractions (spherical power) for the control and calculated IOL groups.

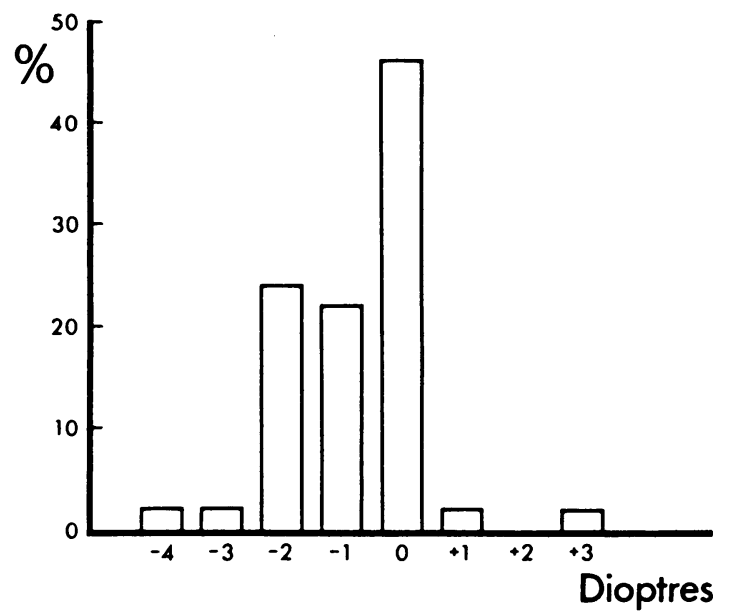

Fig. 4 The differences in dioptres between the calculated predictions of postoperative refraction and the actual postoperative refractions (spherical power) in the calculated group.

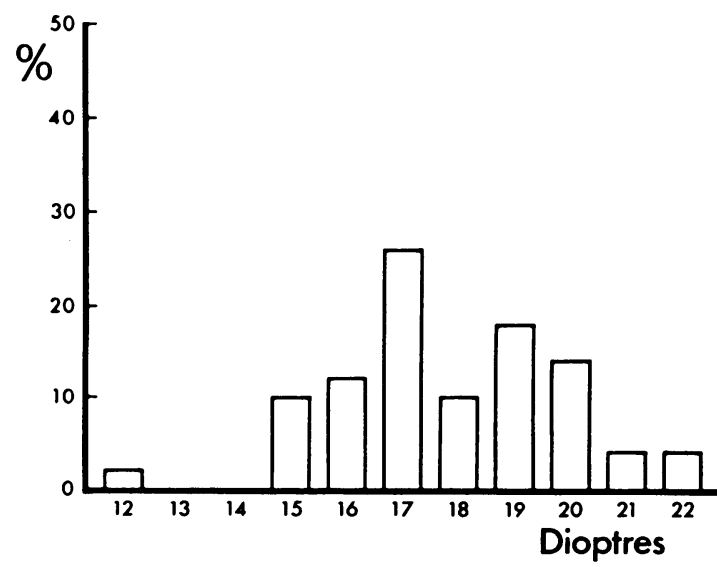

Fig. 5 The powers of IOL (dioptres in aqueous) used in the calculated group after calculation for emmetropia. operative refraction was recorded when the refraction had stabilised and the first postoperative spectacles were prescribed at about the sixth postoperative week.

The postoperative refractions in terms of spherical equivalent (after conversion to plus cylinder form) and spherical power were compared for the control and calculated groups and the accuracy of prediction of postoperative refraction assessed for the calculated group.

\section{Results}

RESIDUAL REFRACTION

The postoperative refractions for the 2 groups are compared in Fig. 2 in terms of spherical equivalent. $70 \%$ of the control eyes were within the $\pm 1 \mathrm{D}$ and $80 \%$ were within the $\pm 2 \mathrm{D}$ range from emmetropia, while $92 \%$ of the calculated eyes were within the \pm 1 $D$ and $98 \%$ within the $\pm 2 \mathrm{D}$ range from emmetropia. For the $\pm 1 \mathrm{D}$ range this difference is statistically significant, with $p<0.01$ by the chi-squared test, and for the $\pm 2 \mathrm{D}$ range this difference is statistically significant with $\mathrm{p}$ abs $\bumpeq 0.0004$ by exact probability testing. The single calculated eye with significant postoperative ametropia (+5 D) was noted preoperatively to have keratometry of doubtful accuracy because of corneal scarring.

Fig. 3 presents the postoperative refractions in terms of spherical power. $47 \%$ of the control eyes were within the $\pm 1 \mathrm{D}$ and $67 \%$ were within the $\pm 2 \mathrm{D}$ range from emmetropia, while $68 \%$ of the calculated eyes were within the $\pm 1 \mathrm{D}$ and $92 \%$ within the $\pm 2 \mathrm{D}$ range from emmetropia.

The mean astigmatism $( \pm S D)$ for the calculated group was $2 \cdot 0 \pm 1 \cdot 4 \mathrm{D}$.

\section{CALCULATED PREDICTION}

The difference between the calculated predictions of postoperative refraction and the actual spherical power is shown in Fig. 4. 70\% of the predictions were within the $\pm 1 \mathrm{D}$ range and $94 \%$ within the $\pm 2 \mathrm{D}$ range from the actual postoperative refraction. 
The distribution of IOL powers used in the calculated group after calculation for emmetropia is shown in Fig. 5, and they ranged from +12 to $+22 \mathrm{D}$.

\section{Discussion}

If one regards a random postoperative refractive error within the $\pm 2 \mathrm{D}$ range of spherical equivalent as acceptable, an IOL of standard $+19 \mathrm{D}$ power leaves $20 \%$ of eyes with ametropia greater than these limits, and some surprisingly large refractive errors are to be expected. The use of IOLs of calculated power almost eliminates significant postoperative ametropia and gives the surgeon control over the postoperative refraction.

In this study the calculated prediction tended to be biased towards hypermetropia with mean error ( \pm SD) of $1.0 \pm 1 \cdot 4 \mathrm{D}$. The $94 \%$ within the $\pm 2 \mathrm{D}$ range compare favourably with the $93 \%$ reported by Kraff et al. ${ }^{3}$ and the $96 \%$ reported by Maloney et al. ${ }^{4}$ within $2 \mathrm{D}$ of prediction, the $97.2 \%$ reported by Johns ${ }^{5}$ within $2.5 \mathrm{D}$, and the $97 \%$ reported by Clevenger ${ }^{6}$ within $3 \mathrm{D}$ of prediction.

There are a number of limitations to the accuracy of IOL calculation and prediction of refraction. ${ }^{1}$ Clinical instruments for the measurement of axial length by ultrasound have an accuracy of about $0 \cdot 1$ $\mathrm{mm}$ and poor technique will reduce this accuracy. Keratometry has an accuracy of about $0.1 \mathrm{~mm}$ and depends on instrument calibration and fixation. The postoperative anterior chamber depth cannot be measured preoperatively and a suitable figure has to be assumed according to the style of IOL implanted. The ultimate effects of errors of these magnitudes on the postoperative refraction are shown in Table 1 and if additive will amount to a spectacle error of about $1 \cdot 0 \mathrm{D}$.

The accuracy of calculation and prediction of refraction is also limited by postoperative astigmatism. Part of the astigmatism is inherent in the corneal curvatures and part is induced by surgery.
Table 1 The effects of errors in axial length, keratometry, and postoperative anterior chamber depth on the final spectacle refraction

\begin{tabular}{ll}
\hline Axial length & $0 \cdot 1 \mathrm{~mm}=0.25 \mathrm{D}$ \\
Keratometry & $0 \cdot 1 \mathrm{~mm}=0.50 \mathrm{D}$ \\
Anterior chamber depth & $0 \cdot 1 \mathrm{~mm}=0.25 \mathrm{D}$ \\
\hline
\end{tabular}

The latter may be minimised but not eliminated by careful surgical technique.

In view of these several limitations there is at present no justification for the use of IOLs in steps of less than $1 \mathrm{D}$ (which is equivalent to about $0.75 \mathrm{D}$ in the spectacle refraction) despite the misleading apparent accuracy of calculations made to several places of decimals.

Biometry and the calculation of IOL power are simple procedures requiring keratometer, ultrasound instrument, and a programmable calculator or access to a computer. The technique carries no hazard to the patient and gives better postoperative refraction results than the implantation of IOLs of standard power. The surgeon has control of the postoperative refraction and can predict and avoid unwanted ametropia.

I thank the Department of Medical Illustration at St James's University Hospital for the preparation of illustrations.

\section{References}

1 Hillman JS. The computer calculation of intraocular lens power -a clinical study. Trans Ophthalmol Soc UK 1980; 100: 222-8.

2 Binkhorst RD. Pitfalls in the determination of intraocular lens power without ultrasound. Ophthalmic Surg 1976; 7: 69-82.

3 Kraff MC, Sanders DR, Lieberman HL. Determination of intraocular lens power: a comparison with and without ultrasound. Ophthalmic Surg 1978; 9: 81-4.

4 Maloney WF, Kratz RP, Mazzocco TR, Davidson B. Posterior chamber intraocular lens power calculation in 441 cases Am IntraOcular Implant Soc J 1979; 5: 349-350.

5 Johns GE. Clinical evaluation of the DBR A-scan unit. Am IntraOcular Implant Soc J 1979; 5: 213-6.

6 Clevenger CE. Clinical prediction versus ultrasound measurement of IOL power. Am Intra-Ocular Implant Soc J 1978; 4: 222-4. 